\title{
Whole-Genome and Segmental Homozygosity Confirm Errors in Meiosis as Etiology of Struma Ovarii
}

\author{
Brittany B. Henderson ${ }^{a}$ Alka Chaubey ${ }^{b}$ Lawrence M. Roth ${ }^{c}$ \\ Stanley J. Robboy ${ }^{d}$ Gregory Tarasidis ${ }^{\text {e }}$ Julie R. Jones ${ }^{\text {b }}$ John M. Sundermann ${ }^{f}$ \\ Jeff Chou ${ }^{g}$ Ann L. Craddock ${ }^{h}$ Leslie Stevenson ${ }^{i}$ Michael J. Friez ${ }^{b}$ \\ Edward H. Kincaid ${ }^{j}$ Roger E. Stevenson ${ }^{i}$ \\ a Division of Endocrinology, Diabetes and Metabolism, Wake Forest University Baptist Medical Center, Winston- \\ Salem, NC, USA; ${ }^{b}$ Division of Diagnostic Laboratories, Greenwood Genetic Center, Greenwood, SC, USA; \\ 'Department of Pathology, Indiana University School of Medicine, Indianapolis, IN, USA; d Department of Pathology, \\ Duke University Medical Center, Durham, NC, USA; ${ }^{e}$ Department of Surgery, Self Regional Healthcare, Greenwood,

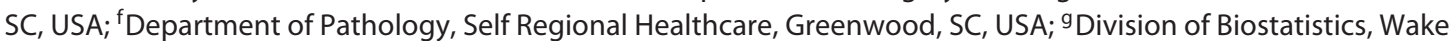 \\ Forest University Baptist Medical Center, Winston-Salem, NC, USA; h Cancer Genomics Shared Resource, Wake Forest \\ University School of Medicine, Winston-Salem, NC, USA; 'Division of Research, JC Self Research Institute, Greenwood \\ Genetic Center, Greenwood, SC, USA; ${ }^{j}$ Division of Cardiothoracic Surgery, Wake Forest University Baptist Medical \\ Center, Winston-Salem, NC, USA
}

\section{Keywords}

Meiosis · Ovary · Struma ovarii · Teratoma · Whole-genome homozygosity

\begin{abstract}
Strumae ovarii are neoplasms composed of normal-appearing thyroid tissue that occur within the ovary and rarely spread to extraovarian sites. A unique case of struma ovarii with widespread dissemination detected 48 years after removal of a pelvic dermoid provided the opportunity to reexamine the molecular nature of this form of neoplasm. One tumor, from the heart, consisting of benign thyroid tissue was found to have whole-genome homozygosity. Another tumor from the right mandible composed of malignantappearing thyroid tissue showed whole-genome homozygosity and a deletion of $7 p$, presumably the second hit that
\end{abstract}

transformed it into a cancerous tumor. Specimens from 2 other cases of extraovarian struma confined to the abdomen and 8 of 9 cases of intraovarian struma showed genomewide segmental homozygosity. These findings confirm errors in meiosis as the origin of struma ovarii. The histological and molecular findings further demonstrate that even when outside the ovary, strumae ovarii can behave nonaggressively until they receive a second hit, thereafter behaving like cancer.

(c) 2019 S. Karger AG, Base

Strumae ovarii are typically benign neoplasms that arise in the ovary and are histologically composed solely or predominantly of normal-appearing thyroid tissue. They are a subtype of mature teratoma, the most common form of

B.B.H. and A.C. contributed equally as first authors. karger@karger.com www.karger.com/cgr

(C) 2019 S. Karger AG, Base

Karger!
Roger E. Stevenson, MD

Division of Research, JC Self Research Institute Greenwood Genetic Center

113 Gregor Mendel Circle, Greenwood, SC 29646 (USA)

E-Mail res@ggc.org 
ovarian neoplasm. Although nearly all strumae ovarii remain confined to the ovary (intraovarian struma, IOS), some can spread to extraovarian sites (EOS) while maintaining an innocuous appearance resembling that of nonneoplastic thyroid. These lesions are exceedingly rare and have been called highly differentiated follicular thyroid carcinoma of ovarian origin, proliferative adenosis, and peritoneal strumosis [Roth and Karseladze, 2008; Robboy et al., 2009; Shaco-Levy et al., 2010, 2012; Hall et al., 2016]. Most EOS tumor deposits that appear histologically benign, although present at remote sites, behave nonaggressively without invasion of surrounding structures [Roth and Karseladze, 2008; Robboy et al., 2009; Shaco-Levy et al., 2010, 2012; Hall et al., 2016]. Alternatively, some strumae ovarii that disseminate appear in both the ovary and extraovarian sites as papillary and follicular carcinomas with typical thyroid cancer histology and marker gene mutations [Schmidt et al., 2007; Coyne and Nikiforov, 2010].

During the past half century, a number of studies using cytogenetic, electrophoretic, and molecular technologies have found evidence for homozygosity at selected loci in some ovarian teratomas but heterozygosity at these loci in the host blood or other tissues [Linder et al., 1975; Parrington et al., 1984; Dahl et al., 1990; Deka et al., 1990; Surti et al., 1990; Kaku et al., 2016; Usui et al., 2018]. A unique case of struma ovarii with widespread dissemination to the face, heart, other internal sites, and skeleton detected 48 years after removal of a pelvic dermoid was found to have whole-genome homozygosity (WG-HMZ). In 2 additional cases of EOS and in 8 of 9 cases of IOS, molecular studies demonstrated genome-wide segmental homozygosity (S-HMZ). These findings confirm the origin of struma ovarii due to errors in meiosis, their capacity for remote dissemination, and their generally benign course unless they undergo a second genetic or genomic hit, thereafter becoming malignant.

\section{Case Reports}

\section{Index Case (EOS 1)}

A 71-year-old white female presented with the history of right temporomandibular joint discomfort recurring for 2 years and right facial swelling for several months. Imaging studies demonstrated a $4.1-\mathrm{cm}$ mass that had destroyed the right mandibular condyle, infiltrated the parotid and masticator space, and involved the right temporomandibular joint. Fine needle aspiration biopsy disclosed a well-differentiated follicular thyroid carcinoma (Fig. 1). Ultrasound demonstrated no suspicious intra-thyroidal lesion or lymphadenopathy. Past medical history was significant for a pelvic dermoid removed 48 years prior without reported malignancy. ${ }^{123} \mathrm{I}$ whole-body scan identified multifocal metastatic struma ovarii that involved the right face, multiple osseous structures, porta hepatis, and interatrial septum of the heart (Fig. 1A, E, G). Cardiac imaging demonstrated a $2.3-\mathrm{cm}$ well-circumscribed mass in the right atrium attached to the interatrial septum near the tricuspid annulus, which subsequently was removed surgically and shown to be bland-appearing follicular thyroid tissue (Fig. 1B-D1). At no time did the patient experience symptoms or laboratory findings of hyperthyroidism. Total thyroidectomy was performed, and histology was negative for thyroid malignancy.

Postoperatively, the patient received $150.2 \mathrm{mCi}^{131} \mathrm{I}$ to treat the disseminated struma together with high-dose prednisone taper to minimize tumor swelling. The right facial pain and swelling slowly resolved. A whole-body scan with ${ }^{123}$ I performed 9 months later showed minimal residual uptake in the mandibular tumor and a $60 \%$ decrease in the mandibular tumor volume (Fig. 1G). Repeat treatment was given with $200 \mathrm{mCi}^{131} \mathrm{I}$, and no uptake at any of the tumor sites could be detected 18 months later.

Other Cases (EOS 2 and 3, IOS 1-9)

Subsequent to molecular study of the index case (EOS 1), archived tissues (formalin-fixed, paraffin-embedded, FFPE) from 2 additional cases of EOS and 9 cases of IOS were acquired for study. Specimen EOS 2 was a para-aortic lymph node containing benignappearing thyroid tissue which had been removed from a 58-yearold woman 26 years after excision of ovarian struma. Specimen EOS 3 was a peritoneal tumor from a 31-year-old woman, 9 years after right oophorectomy for an $8-\mathrm{cm}$ struma ovarii. The 9 IOS specimens were obtained surgically before recognized spread to extraovarian tissues.

\section{Materials and Methods}

\section{Samples and Histological Validation}

Peripheral blood, normal thyroid tissue, and tumor tissue from the heart and right mandible were obtained from the index patient (EOS 1) prior to radioactive iodine therapy. FFPE tissues from additional EOS tumors were obtained from Indiana University and Duke University (EOS 2, EOS 3), and 9 cases of IOS tumors were obtained from Wake Forest Baptist Medical Center. Microtome slices of the FFPE tissue blocks were manually cleared of surrounding paraffin and DNA isolated from the remaining tissue without microdissection. Slides of the FFPE tumors were stained with hematoxylin and eosin for histological examination.

\section{OncoScan ${ }^{T M}$ Microarray Analysis}

DNA was isolated from frozen cardiac tumor, aspirates of the mandibular tumor, FFPE thyroid tissue, and host blood from the index case and from FFPE tissues from EOS 2 and 3 and IOS 1-9 tumors using standard laboratory procedures [Gliem and Aypar, 2017; Louie et al., 2017]. The isolated DNA was analyzed using the Applied Biosystems OncoScan ${ }^{\mathrm{TM}}$ microarray system (Thermo Fisher Scientific, San Francisco, CA). SNP genotyping on this platform has the enhanced ability to identify long contiguous stretches of homozygosity and uniparental disomy as well as targeted genetic mutations (e.g., $B R A F^{\mathrm{V} 600 \mathrm{E}}$ ). Chromosome Analysis Suite (ChAS) software v.3.3 was utilized for analysis. All copy number changes were determined using the human genome build 19 (hg19/NCBI build 37). 

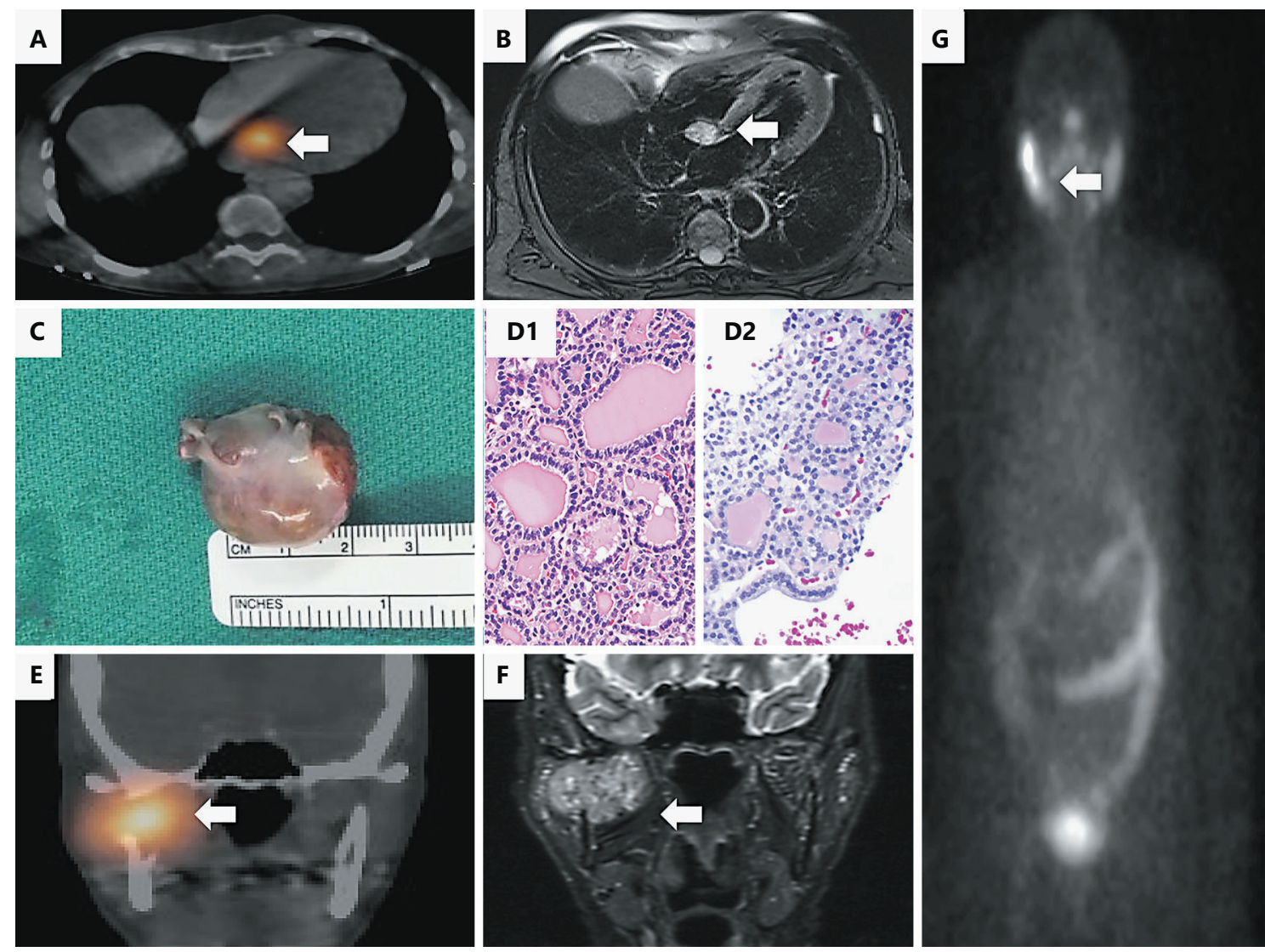

Fig. 1. Index case EOS 1. Imaging and histology of tumors. A Axial SPECT/CT shows ${ }^{123} \mathrm{I}$ uptake in the region of the right atrium (arrow) concerning for metastasis. B Axial cardiac MRI shows the heterogeneous, T2-weighted hyperintense mass (arrow) adherent to the interatrial septum. C A 2.3-cm well-circumscribed mass was removed surgically from the right atrium. D1 Histology of the cardiac tumor demonstrating bland-appearing follicular thyroid tissue without invasion into surrounding structures. D2 Histology of an aspirate of the mandibular tumor, showing round, uniform,

Whole-Exome Sequencing

Whole-exome sequencing (WES) was performed on DNA from the index patient's blood and frozen cardiac tumor to comprehensively evaluate for the common thyroid cancer-associated gene alterations. Methods for DNA isolation followed standard laboratory procedures [Gliem and Aypar, 2017; Louie et al., 2017]. Mutation candidates were selected based on findings of the Cancer Genome Atlas Research Network [2014].

RNA Sequencing

RNA was extracted from FFPE slides from the cardiac tumor of EOS 1, the para-aortic lymph node of EOS 2, and a peritoneal tumor of EOS 3 by standard protocols [Kukurba and Montgomery, 2015]. RNA analysis was performed after sequencing to a read depth of 130 million reads per sample using paired-end sequencing with Mid Output 150-cycle kit (Illumina Inc.) on the Illumina NextSeq 500. Gene fusion analysis was performed using fusionCatcher and 3 aligners: Bowtie, blat, and STAR.

and slightly overlapping nuclei forming small follicles, some of which contained colloid. E Coronal ${ }^{123}$ I SPECT/CT of the head shows uptake in the soft tissue surrounding the bone. $\mathbf{F}$ Coronal STIR MRI shows a heterogeneous mass in the right mandible, corresponding to radioactive iodine uptake. G Anterior ${ }^{123} \mathrm{I}$ wholebody scan several months after initial radioactive iodine treatment shows mild residual uptake in the region of the right maxilla (arrow), likely residual disease. Subsequently, the patient was retreated with $200 \mathrm{mCi}^{131} \mathrm{I}$.

\section{Results}

\section{Gross and Histological Analysis}

On gross examination, the tumor from the interatrial septum consisted of an oval nodule partly covered by glistening membrane (Fig. 1C). Microscopic examination was performed on the index patient's mandibular and cardiac tumors for comparison with histology of the patient's thyroid gland. Histological assessment of the interatrial tumor demonstrated bland thyroid tissue (Fig. 1D1). Histological examination of aspiration biopsies of the mandibular tumor showed thyroid microfollicles with a single cell lining. The cells showed nuclei that were slightly overlapping, round, and uniform, with only rare mitotic figures (Fig. 1D2). There were no nuclear features of 

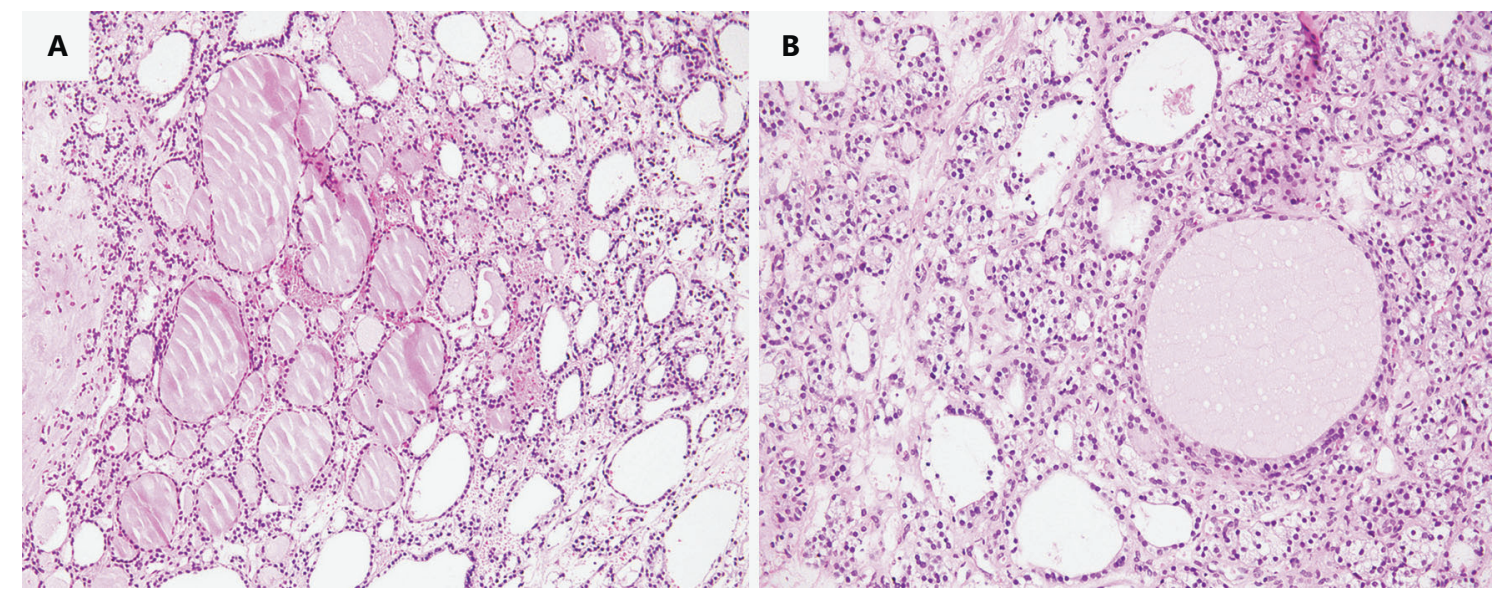

Fig. 2. Histology of EOS 3. A The specimen from the local recurrence of struma ovarii shows a mixture of small and large thyroid-type follicles filled with colloid. B A section from a subsequent peritoneal recurrence shows solid clusters of follicular cells devoid of colloid, highly suggestive of progression of the neoplasm along with residual small and large thyroid-type follicles.

papillary carcinoma. Immunohistochemical stains for thyroid transcription factor 1 and thyroglobulin showed positivity in the nuclei and cytoplasm, respectively, confirming thyroid differentiation. The patient's thyroid gland demonstrated normal thyroid histology.

Histological examination of tissues from EOS 2 showed peritoneal and omental nodules consisting of foci of thyroid follicles of variable size resembling a colloid nodule of the thyroid gland. In a few small areas, microfollicles predominated. The nuclei were uniform and normochromatic. Nuclear features of thyroid-type papillary carcinoma were absent. Mitoses were not identified. The paraappendiceal fat and the region of the cystic duct contained similar tumor nodules. A para-aortic lymph node was partially replaced by similar neoplasm. The cervical thyroid gland was histologically normal except for a small colloid nodule. Sections of the primary left ovarian neoplasm were unavailable.

Histological examination of the local recurrence from EOS 3 showed nodular thyroid-type tissue composed of clusters of small and large follicles that resembled a colloid nodule of the thyroid gland. No significant atypia was identified (Fig. 2A). In the late recurrences, the histology of EOS 3 evolved to a higher follicular cell density with loss of follicular colloid together with a few residual thyroid-type follicles of variable size (Fig. 2B).

The 9 cases of IOS, including IOS 9 which showed heterozygosity across the entire genome, demonstrated benign appearing thyroid tissue without histological evidence of malignant transformation.

Meiotic Errors as Etiology of Struma Ovarii

\section{Genomic Analyses}

The OncoScan SNP microarray, which assesses the molecular constitution of tumor and nontumor tissues, can clearly show the genome's copy number, the existence of allelic homozygosity or heterozygosity and, in some cases, the location and number of crossovers that occur on each chromosome. Microarray studies on the index case demonstrated normal heterozygosity in the peripheral blood and thyroid gland but WG-HMZ in her cardiac and mandibular tumors (Fig. 3). The atrial tumor, which was not invasive and only adherent to the atrial wall, demonstrated WG-HMZ only, whereas the aggressively behaving mandibular tumor showed both WG-HMZ and a 13.8-Mb deletion of chromosome $7 \mathrm{p} 15.3 \mathrm{p} 14.1$. In both the atrial and mandibular samples, the presence of nontumor supporting tissue from the host appears as a minor population with heterozygosity in the OncoScan images (Fig. 3B, C).

EOS 2 and 3 and all but one of the IOS samples demonstrated varying degrees of S-HMZ interspersed with segments of heterozygosity (Fig. 4, 5). The degree of SHMZ was similar among IOS and EOS tumors. Although S-HMZ usually involved chromosomal segments located close to the centromere and segments beyond the second crossover on the arms of certain chromosomes, 2 cases were heterozygous in the pericentromeric region (Fig. 5A, C). Chimerism appeared in all tumor samples except IOS 9, presumably reflecting the presence of nontumor supporting tissues having the patients' constitutional genomes (Fig. 3B, C, 4, 5). A ready explanation for hetero- 


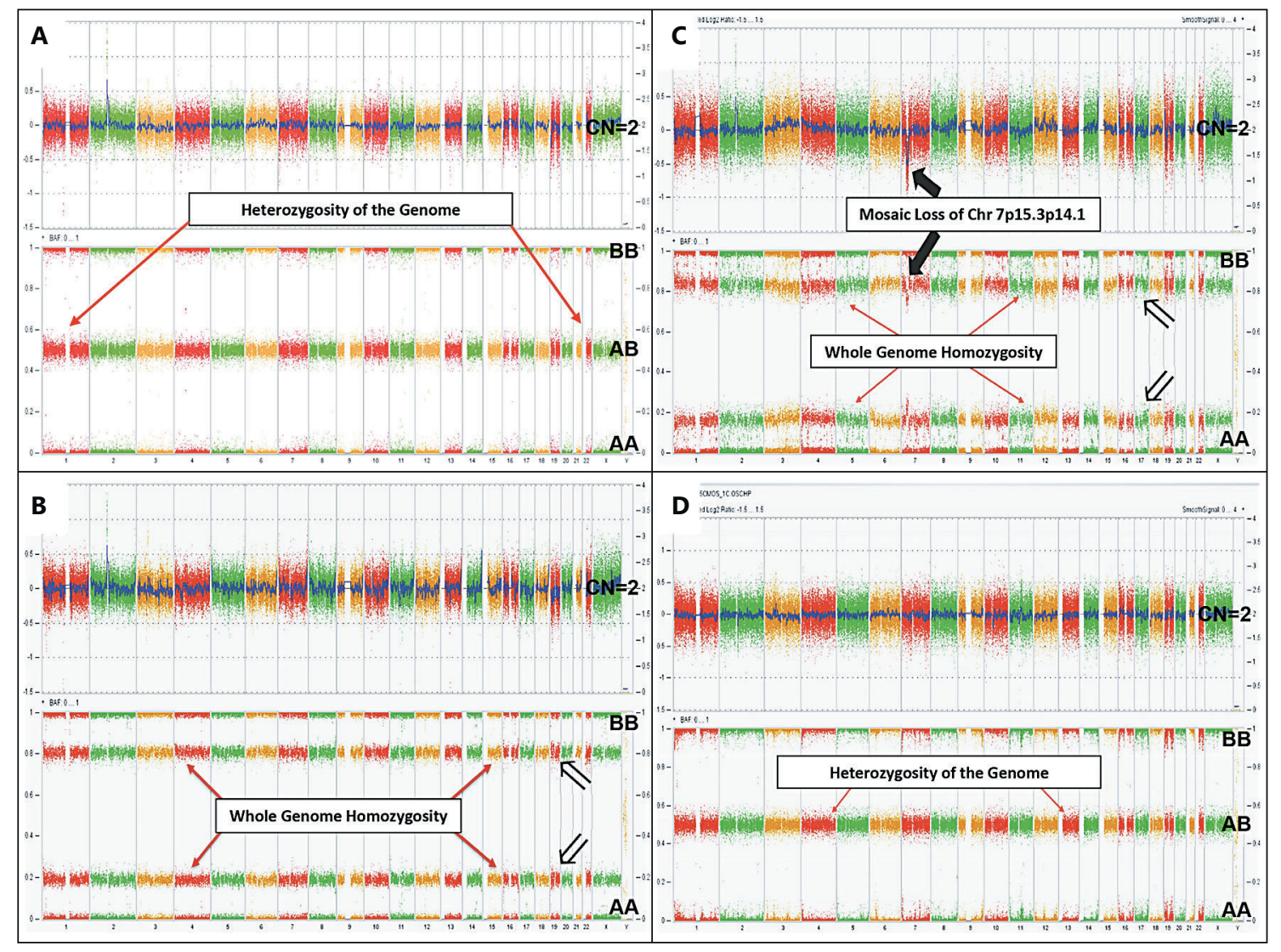

Fig. 3. Index case EOS 1 . Whole-genome OncoScan SNP microarray profiles. A DNA from blood. Top panel: Normal copy number state (blue line; $\mathrm{CN}=2$ ) is shown by the Log $\mathrm{R}$ ratio plot (copy number probes for each chromosome are shown as dots) across the genome. Bottom panel: a normal B-allele frequency (BAF) plot demonstrates heterozygosity across the genome. The SNP probes are plotted in the ChAS software and reflect the number of $\mathrm{B}$ alleles in comparison to the total number of alleles (A and B). The $\mathrm{BB}$ homozygote is reflected by a BAF of 1.0 and the AA homozygote is reflected by the $\mathrm{BAF}$ of 0.0 , whereas the $\mathrm{AB}$ heterozygote represents the $\mathrm{BAF}$ of 0.5 . Chromosomes 1 through $\mathrm{X}$ are shown in different colors from left to right. B DNA from atrial tissue, showing a normal copy state (blue line in the $\log _{2}$ ratio; $\mathrm{CN}=2$ ) across the genome. The BAF demonstrates whole-genome homo-

zygosity of the entire genome in IOS 9 (Fig. 5I) cannot be given, but suggests that the DNA was isolated from supporting tissues from the host rather than from the tumor.

WG-HMZ or S-HMZ found in the EOS tumors and all but one of the IOS tumors was considered to be the primary genomic alteration. Homozygosity alone appeared responsible for intra-abdominal and remote tumor dissemination of EOS 1 and EOS 2. Three chromosome gains were present in EOS 3. None of the typical thyroid cancer gene mutations or fusions reported by the Cancer zygosity (WG-HMZ) appearing as absence of heterozygosity across the genome. The presence of nontumor supporting tissue from the host genome is indicated by open arrows in the lower BAF plot. C DNA from the mandibular tumor shows a similar OncoScan profile of WG-HMZ as the cardiac tumor. Also, a mosaic loss of a 13.8-Mb region in chromosome $7 \mathrm{p}$, arr[hg19] 7p15.3p14.1(23523742_37323278) $\times 1 \sim 2$, was identified in this tissue specimen (black arrows). This is indicative of an admixture of 2 cell lines (affected tumor and normal connective tissue). D DNA from the thyroid gland demonstrating a normal genomic dosage and profile as in the blood. Note the absence of the restricted copy number gain near the centromere of chromosome 2 , which is present in the patient's blood (A), atrial tissue (B), and mandibular tumor (C), a finding that is not explained.

Genome Atlas Research Network [2014] was observed on whole-genome sequencing of the cardiac tumor from EOS 1 or RNA sequencing of the tumors EOS 1-3. In EOS 1 , the index case, there was mosaic loss of $13.8 \mathrm{Mb}$ of the short arm of chromosome 7 in the patient's mandibular tumor:arr[hg19]7p15.3p14.1(23523742_37323278) ×1 2 including a total of 173 genes, of which 69 had OMIM entries, notably HOXA genes $1-11$ and 13 . The EOS 3 tumor demonstrated whole-chromosome gains of chromosomes 3, 20, and X (Fig. 4B). 


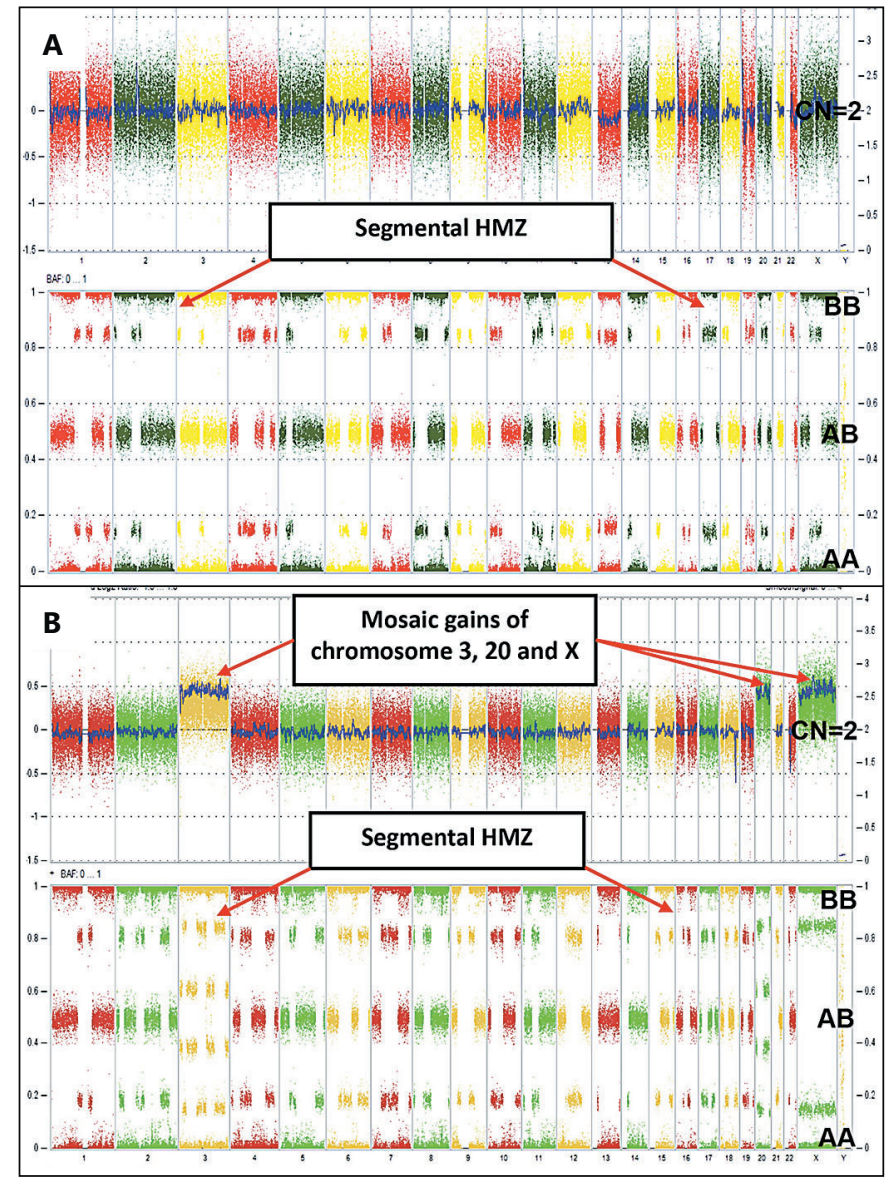

Fig. 4. Whole-genome OncoScan SNP microarray profiles from EOS 2 (A) and EOS 3 (B) demonstrating segmental homozygosity interspersed with regions of heterozygosity in every chromosome. Homozygosity is evident in chromosomal segments closest to the centromere, and the segment telomeric to a second cross-over event in certain chromosomes. Heterozygosity is evident in the intervening segments across the genome in both EOS 2 and EOS 3. Mosaic gains of chromosomes 3, 20, and X were identified in EOS 3.

\section{Discussion}

The inaugural studies on the origin of ovarian teratomas by Linder et al. [1975] using juxtacentromeric chromosome polymorphisms and more distant electrophoretic markers concluded that benign teratomas arose after meiosis I from single germ cells. Seventeen chromosome polymorphisms were uniformly homozygous in the tumors, whereas the host tissues were heterozygous. Their electrophoretic studies gave a different result with only half of the tumors being homozygous. Of the possibilities they considered, only the failure of the second meiotic division or fusion of the second polar body with its ootid was consistent with their observations.

Subsequent studies spanning the next 4 decades using cytogenetic, electrophoretic, and molecular technologies found that some ovarian teratomas were homozygous at all loci examined, while others were heterozygous at all loci, and still others showed a mixture of homozygosity and heterozygosity at different loci [Parrington et al., 1984; Dahl et al., 1990; Deka et al., 1990; Surti et al., 1990; Kaku et al., 2016; Snir et al., 2017; Kato et al., 2018; Usui et al., 2018]. Surti et al. [1990] and Deka et al. [1990], in reviewing the data from multiple studies, identified the 5 mechanisms that could explain the origin of ovarian teratomas:

(1) Failure of an oogonium to undergo meiosis (premeiotic origin)

(2) Failure of meiosis I or fusion of the primary oocyte with its polar body

(3) Failure of meiosis II or fusion of the secondary oocyte with its polar body

(4) Endoreduplication of the haploid genome of a mature ovum

(5) Fusion of 2 ova (autofertilization)

Of these 5 mechanisms, only 3 (2, 3, and 4) have supporting evidence. Four recent molecular studies are especially notable [Kaku et al., 2016; Snir et al., 2017; Kato et al., 2018; Usui et al., 2018]. Kaku et al. [2016] did not find evidence for the first mechanism (premeiotic origin) among 156 mature cystic teratomas. Usui et al. [2018], using SNP microarray data from 38 mature cystic teratomas, found evidence for meiosis I errors, meiosis II errors, and endoreduplication of mature ova, but no evidence for a premeiotic error (mechanism 1) or for fusion of the haploid genomes of 2 different ova (mechanism 5). Kato et al. [2018], who investigated the genetic zygosity of all forms of ovarian teratomas, found homozygosity in 3 of 6 (50\%) informative struma ovarii samples. WG-HMZ of some tumors was reported in these studies, but included no information about dissemination [Kaku et al., 2016; Kato et al., 2018; Usui et al., 2018]. Snir et al. [2017] found extraovarian dissemination but no recurrences among 7 immature teratomas ( 2 with WG-HMZ and 4 with S-HMZ), extraovarian dissemination and recurrences among 6 mixed germ cell tumors (1 with nearly complete HMZ and 2 with SHMZ), and absence of extraovarian dissemination or recurrence among 8 mature teratomas ( 2 with WG-HMZ and 3 with S-HMZ).

Endoreduplication of the haploid genome of a mature ovum is perhaps the mechanism most acceptable to ex- 


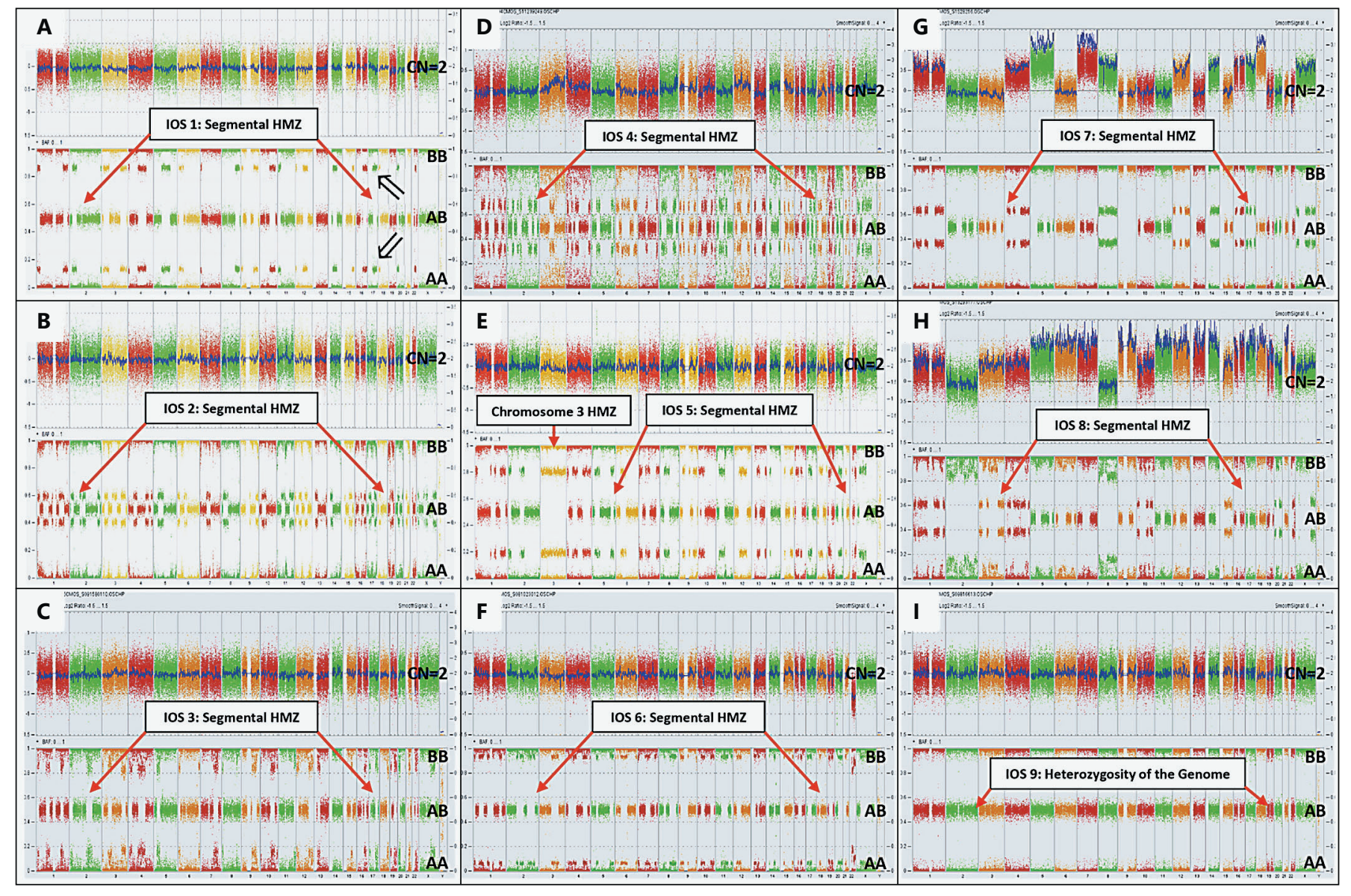

Fig. 5. OncoScan SNP microarray profiles for 9 IOS tumors. A-D IOS 1-4 show segmental homozygosity (S-HMZ) without other chromosome gains or losses. E IOS 5 shows S-HMZ for all chromosomes except chromosome 3. F-H IOS 6-8 show S-HMZ with one or more chromosome gains or losses suggesting possible transition toward malignancy. A loss of chromosome 22 is present in

plain WG-HMZ in the index case EOS 1. WG-HMZ could also occur if the haploid genome of the second polar body underwent endoreduplication and then proliferated. Other mechanisms leading to WG-HMZ would require 2 prezygotic errors (e.g., failure of chiasma formation in meiosis I coupled with endoreduplication after meiosis II). EOS 2, EOS 3, and 8 of the 9 IOS tumors demonstrated crossovers confirming that chiasmata formed and crossovers occurred in every chromosome and in usual numbers in meiosis I (70-95 per cell in females) [Tease and Hultén, 2004]. Among these 10 tumors, 2 (IOS 1 and IOS 3) were heterozygous at one or more centromeres, indicating a failure of meiosis I or fusion of a primary oocyte with its polar body. The other tumors were homozygous at the centromere, indicating a
IOS $6(\mathbf{F})$ and multiple chromosome gains are present in IOS 7 and IOS $8(\mathbf{G}, \mathbf{H})$. Note in all these cases $(\mathbf{A}-\mathbf{H})$ the presence of chimerism from the host genome appearing as a minor population of whole-genome heterozygosity (about $15 \%$ in A, $40 \%$ in B, $15 \%$ in C, etc.) as indicated by open arrows in A. I IOS 9 shows normal whole-genome heterozygosity. meiosis II error or fusion of the secondary oocyte with its polar body.

None of the tumors, IOS or EOS, harbored the typical thyroid cancer driver mutations or gene fusions, such as $B R A F^{\mathrm{V} 600 \mathrm{E}}$ or $R A S$, seen in some cases of malignant struma ovarii [Schmidt et al., 2007; Coyne and Nikiforov, 2010; Cancer Genome Atlas Research Network, 2014]. Instead, it appears that homozygosity of the genome, either complete or segmental, was the predominant genetic aberration. It is unclear whether the type of homozygosity (whole genome or segmental), or the timing of the prezygotic error, influences the propensity to spread to extraovarian sites or to undergo malignant transformation [Tokuda et al., 1993; McDougall, 2006; Ruel et al., 2010; Carey et al., 2014]. It does not escape attention that 
the case with the most widespread dissemination and the longest latent period before detection (EOS 1) was associated with WG-HMZ. The aggressive nature of the mandibular tumor in this case may have been caused by the $13.8-\mathrm{Mb}$ copy loss of 7p15.3p14.1 involving 173 genes, presumably its "second hit." Within the deleted region were the HOXA genes (1-11, and 13), primarily involved in body axis patterning, stem cell renewal, cell fate, and cell differentiation in many tissue types including the thyroid gland. Mutations in HOX family genes have been reported to play a role in carcinogenesis and in thyroid tumor aggressiveness [Takahashi et al., 2004; Bhatlekar et al., 2014]. Specifically, HOX is an important regulator of E-cadherin, with loss promoting tumor aggressiveness, invasion, and tumor migration [Bhatlekar et al., 2014]. Additionally, EOS 3 demonstrated several mosaic wholechromosome gains of chromosomes 3, 20, and X. Histological assessment of EOS 3 and multiple abdominal recurrences demonstrated evolution to a more aggressive pathology (i.e., loss of normal follicles) with time, again supporting the "second hit" hypothesis. Alternatively, the chromosome gains could have occurred concurrently with the meiotic error and had no influence on the evolution of the histological appearance.

The OncoScan microarray demonstrated that 8 of the 9 IOS and the 3 EOS tumors in this study have similar molecular profiles, most commonly S-HMZ, but in 1 case WG-HMZ. These molecular profiles confirm that monodermal teratomas with struma ovarii histology arise in the prezygotic state from errors in meiosis I and/or II and in this regard are similar to all ovarian teratomas. Our data suggest that EOS tumors without second hits appear molecularly identical to IOS tumors without invasion of adjacent tissue; conversely, those with second hits behave more aggressively. Homozygosity possibly predisposes these tumors to significant genetic mutations, chromosomal gains or losses, and gene fusions that may contribute to tumor aggressiveness and malignant behavior. Absence of typical thyroid cancer gene mutations or fusions in EOS 1 suggests that WG-HMZ may be the primary genetic alteration associated with dissemination beyond the abdomen, a finding that may have implications for other tumor types. Among the other literature cases with WG-HMZ, clinical details regarding dissemination and aggressiveness of the tumors were provided only in the report by Snir et al. [2017]. Prior to the availability of molecular studies, a number of instances were reported in which struma spread beyond the peritoneal cavity to the lungs, liver, brain, vertebrae, cranium, pelvic bones, ribs, and long bones [Tokuda et al., 1993; McDougall, 2006;
Ruel et al., 2010; Carey et al., 2014]. Among these, 1 case with dissemination to the liver was detected 40 years after removal of "tumors" of the ovary [McDougall, 2006]. Dissemination to the heart may be unique to the index case EOS 1 presented here.

The course of the index case EOS 1 challenges the concept that extraovarian spread of struma ovarii should be considered malignant. Struma spread to the heart, lungs, porta hepatis, other soft tissues, spine, and ribs followed a benign course even decades after removal of the primary pelvic dermoid. Only with the second hit, a deletion involving chromosome $7 \mathrm{p}$, did a tumor in the right face become malignant, invading the mandible. The OncoScan microarray should provide an effective technology to distinguish primary thyroid carcinoma from carcinoma arising in struma ovarii.

\section{Acknowledgements}

We gratefully acknowledge the assistance and advice from Drs. Barbara DuPont and Timothy Fee and from Thermo Fisher Scientific, San Francisco, CA.

\section{Statement of Ethics}

Written informed consent was given by all subjects for publication. The Wake Forest Baptist University Medical Center Institutional Review Board (IRB) approved the collaborative study as did the IRBs at Indiana University and Duke University.

\section{Disclosure Statement}

The authors have no conflicts of interest to declare.

\section{Funding Sources}

The authors would like to acknowledge support from grants P30 CA012197-39 and AN 3932506. Additional support was provided by the Greenwood Genetic Center Foundation and by the Cancer Genomics Shared Resource through the Wake Forest Baptist Comprehensive Cancer Center's NCI Cancer Center Support Grant P30CA012197.

\section{Author Contributions}

All authors made substantial contributions to this work and have given final approval for this version to be published. The authors confirm its accuracy and integrity and agree to be accountable for all other aspects of this work. 


\section{References}

- Bhatlekar S, Fields JZ, Boman BM: HOX genes and their role in the development of human cancers. J Mol Med (Berl) 92:811-823 (2014).

-Cancer Genome Atlas Research Network: Integrated genomic characterization of papillary thyroid carcinoma. Cell 159:676-690 (2014).

Carey K, Jain M, Krishna M, Accurso J: Peritoneal and mediastinal highly differentiated follicular carcinoma of ovarian origin. Indian J Nucl Med 29:108-111 (2014).

-Coyne C, Nikiforov YE: RAS mutation-positive follicular variant of papillary thyroid carcinoma arising in a struma ovarii. Endocr Pathol 21:144-147 (2010).

Dahl N, Gustavson KH, Rune C, Gustavsson I, Pettersson U: Benign ovarian teratomas. An analysis of their cellular origin. Cancer Genet Cytogenet 46:115-123 (1990).

-Deka R, Chakravarti A, Surti U, Hauselman E, Reefer J, et al: Genetics and biology of human ovarian teratomas. II. Molecular analysis of origin of nondisjunction and gene-centromere mapping of chromosome I markers. Am J Hum Genet 47:644-655 (1990).

-Gliem TJ, Aypar U: Development of a chromosomal microarray test for the detection of abnormalities in formalin-fixed, paraffin-embedded products of conception specimens. J Mol Diagn 19:843-847 (2017).

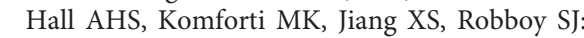
Thyroid type carcinomas of ovarian origin (malignant struma ovarii). AJSP: Reviews \& Reports 21:227-231 (2016).

Kaku H, Usui H, Qu J, Shozu M: Mature cystic teratomas arise from meiotic oocytes, but not from pre-meiotic oogonia. Genes Chromosomes Cancer 55:355-364 (2016).

Kato N, Sakamoto K, Murakami K, Iwasaki Y, Kamataki A, Kurose A: Genetic zygosity of mature ovarian teratomas, struma ovarii, and ovarian carcinoids. Virchows Arch 473:177182 (2018).
Kukurba KR, Montgomery SB: RNA sequencing and analysis. Cold Spring Harb Protoc 2015: 951-969 (2015).

Linder D, McCaw BK, Hecht F: Parthenogenic origin of benign ovarian teratomas. $\mathrm{N}$ Engl J Med 292:63-66 (1975)

Louie RJ, Tan QK, Gilner JB, Rogers RC, Younge $\mathrm{N}$, et al: Novel pathogenic variants in FOXP3 in fetuses with echogenic bowel and skin desquamation identified by ultrasound. Am J Med Genet A 173:1219-1225 (2017).

McDougall IR: Metastatic struma ovarii: the burden of truth. Clin Nucl Med 31:321-324 (2006).

$>$ Parrington JM, West LF, Povey S: The origin of ovarian teratomas. J Med Genet 21:4-12 (1984).

Robboy SJ, Shaco-Levy R, Peng RY, Snyder MJ, Donahue J, et al: Malignant struma ovarii: an analysis of 88 cases, including 27 with extraovarian spread. Int J Gynecol Pathol 28:405422 (2009).

Roth LM, Karseladze AI: Highly differentiated follicular carcinoma arising from struma ovarii: a report of 3 cases, a review of the literature, and a reassessment of so-called peritoneal strumosis. Int J Gynecol Pathol 27:213-222 (2008).

Ruel IF, Fierrard H, Vercellino L, Bernard L, Hindie E, et al: Pulmonary metastasis of struma ovarii: a case report. Clin Nucl Med 35: 692-694 (2010).

Schmidt J, Derr V, Heinrich MC, Crum CP Fletcher JA, et al: $B R A F$ in papillary thyroid carcinoma of ovary (struma ovarii). Am J Surg Pathol 31:1337-1343 (2007).

Shaco-Levy R, Bean SM, Bentley RC, Robboy SJ: Natural history of biologically malignant struma ovarii: analysis of 27 cases with extraovarian spread. Int J Gynecol Pathol 29:212227 (2010).
Shaco-Levy R, Peng RY, Snyder MJ, Osmond GW, Veras E, et al: Malignant struma ovarii: a blinded study of 86 cases assessing which histologic features correlate with aggressive clinical behavior. Arch Path Lab Med 136: 172-178 (2012).

- Snir OL, DeJoseph M, Wong S, Buza N, Hui P: Frequent homozygosity in both mature and immature ovarian teratomas: a shared genetic basis of tumorigenesis. Mod Pathol 30:14671475 (2017).

-Surti U, Hoffner L, Chakravarti A, Ferrell RE: Genetics and biology of human ovarian teratomas. I. Cytogenetic analysis and mechanism of origin. Am J Hum Genet 47:635-643 (1990).

Takahashi Y, Hamada J, Murakawa K, Takada M, Tada M, et al: Expression profiles of 39 HOX genes in normal human adult organs and anaplastic thyroid cancer cell lines by quantitative real-time RT-PCR system. Exp Cell Res 293:144-153 (2004).

Tease C, Hultén MA: Inter-sex variation in synaptonemal complex lengths largely determine the different recombination rates in male and female germ cells. Cytogenet Genome Res 107:208-215 (2004).

Tokuda Y, Hatayama T, Sakoda K: Metastasis of malignant struma ovarii to the cranial vault during pregnancy. Neurosurgery 33:515-518 (1993).

Usui H, Nakabayashi K, Kaku H, Maehara K, Hata K, Shozu M: Elucidation of the developmental mechanism of ovarian mature cystic teratomas using B allele-frequency plots of single nucleotide polymorphism array data. Genes Chromosomes Cancer 57:409-419 (2018). 